\title{
Mobile Haptic Technology Development through Artistic Exploration
}

\author{
David Cuartielles ${ }^{1}$, Andreas Göransson ${ }^{2}$, Tony Olsson ${ }^{2}$, and Ståle Stenslie ${ }^{3}$ \\ ${ }^{1}$ Medea, Malmö University, Sweden \\ david.cuartielles@mah.se \\ ${ }^{2}$ K3 - Malmö University, Sweden \\ \{andreas.goransson, tony.olsson\} @mah.se \\ ${ }^{3}$ Faculty of Humanities, Aalborg University, Denmark \\ stenslie@hum.aau.dk
}

\begin{abstract}
This paper investigates how artistic explorations can be useful for the development of mobile haptic technology. It presents an alternative framework of design for wearable haptics that contributes to the building of haptic communities outside specialized research contexts. The paper also presents our various wearable haptic systems for mobile computing capable of producing high-order tactile percepts. Our practice based approach suggests a design framework that can be applied to create advanced haptic stimulations/situations for physically embodied interaction in real-world settings.
\end{abstract}

Keywords: Applied haptics, wearables, bodysuit, haptic and embodied interaction, haptic resolution, Arduino, Android, mobile haptic systems, online haptics editor.

\section{$1 \quad$ Introduction}

This paper presents several of our artistic developments using mobile haptic technology with multiple tactile outputs (16+). These represent low cost, open-source haptic systems that use off the shelf components. Our approach intends to act as toolsets for designers working with haptic systems that create emotional and immersive haptic experiences. Rather than developing customized systems aimed at specific tasks or purposes we have made a set of "modules" that are tied together with a shared communication protocol. This approach allows for quick high-fidelity prototype development and faster, simplified iterations of the design. Since our projects are primarily based on standard components, they can be developed at a low start-up cost and propagate reusability.

Our projects have progressed in a chain of iterated design processes where the hardware and the conceptual components have affected each other. The conceptual content part of the system is based on an experimental media art approach where the goal is to create a multisensory, immersive and embodied experience system centered on an open exploration of a poetics of touch. The term embodiment is here understood as a combination of both a physical presence in the world and a social embedding in a 
web of practices and purposes [1]. The resulting systems have been successfully tried at several usability tests during public art events in Norway, Sweden, Denmark and Slovenia.

\subsection{Haptic Systems History}

In history the concept of haptic communication through cutaneous touch can be traced back to Giovanni Battista della Porta who in 1558 described the sympathetic telegraph [2]. His proposal was to use magnetism to send and receive the same message over distance, encrypting and decrypting messages by tapping on to the body of two users. This rather imaginative device has never been built, but the concept represents an interesting first approach towards personal, direct and embodied corporal connectivity.

Other early concepts involving touch was Edison's 'Telephonoscope' [3] which preconceived a telepresence system much like the later videoconferencing systems of today. An important inspiration for telepresence is the notion of being present at the other end of the communication line, as if one was physically present, sensing and interacting with one's own body.

One early important work on tactile interface technology was Bach-y-Rita's first 'tactile display' built in the 1960s [4]. A 20-by-20 array of metal rods in the back of chair were wired to act as the pixels of a screen and functioned much like an electronic Braille writer continuously raising and lowering 'dots' recognizable by the tactile senses. With this tactile display people sitting in the chairs could identify 'pictures' as they were poked into their backs. In effect this demonstrates cross modal perception, allowing us to see images with our sense of touch [5].

Creating a sense of tactile immersion through tactile manipulation of the senses is still difficult to invoke. Current haptic systems within areas such as telemedicine, telerobotics, $\mathrm{CAD} / \mathrm{CAM}$ and virtual reality are primarily desktop based using technologies such as the PHANToM ${ }^{1}$ (Personal HAptic iNTerface Mechanism) [5] and haptic gloves [6]. Most haptic interaction systems are based on a desktop paradigm [7]. This also goes for high resolution wearable displays such as the Tactile Torso Displays [8]. In our world of emerging smartphone- and mobile computing for users on the move, we foresee the need of wearable systems with a higher degree of mobility.

\subsection{Towards Mobile and Wearable Haptic Systems}

Today there exist no standard or commercially available systems for complex, high resolution haptic interfaces dedicated to mobile and wearable use. At the same time users are adapting to simple haptic systems such as vibrating screens and mobile phones, indicating both the growing need and possibility for somatosensory and haptic systems in communications and experience design. In later years wearable computing has become an extension of ubiquitous computing. This post-desktop, user-centric paradigm of human-computer-interaction focuses on embedding computational power

1 http: / /www. sensable.com/products-haptic-devices.htm accessed on June 7, 2012. 
seamlessly in everyday objects [9]. York also refers to it as machine fitting into the human environment [10]. By fitting haptic technology onto and into our bodies we can provide mobile users with information that was previously unavailable [11], one example being site-specific information and sensing related to users position (GPS) and orientation. For example the 'FeelSpace' belt enables its user to feel his/her orientation in space via vibrotactile stimulation [12]. Such wearable systems are within a wearer's intimacy zone and therefore also have the potential to provide novel and highly expressive forms of interactions. An early example of wearable, haptic bodysuits, albeit attached to desktop computers, is the 'cyberSM' system from 1993 [13] that connects two users over the internet allowing them to see, hear and touch each other.

One of the first mobile and telehaptic art projects was the 'Mobile Feelings' project (2002-03) by Christa Sommerer \& Laurent Mignonneau [14]. Here two users each held an egg shaped communication interface that let the users exchange heartbeats. The haptic effect was created with only one vibratory output, but still let users 'feel a strong sensation of bodily connection' [15]. They also note that 'the sense of touch still remains one of our most private sensation for which we still lack a concise language to describe'. However, as a language of touch appears contingent on haptic resolution ${ }^{2}$ [13], it is likely that the minimal haptic resolution of one vibrator influenced the lack of haptic expressivity.

The 'Hug Shirt' by the CuteCircuit company [16] attempts to construct haptic communication for simple, personal messages between users wearing what appears to be a normal looking shirt. The shirt transmits 'hugs' to another, similar shirt via a Bluetooth and Java enabled telephone device. The stimulus resembling a hug is produced by vibrotactile stimulation. Although scarcely described the shirt apparently has a haptic resolution of 10+ effectors. The company has worked on developing a taxonomy of hugs, but its effects are unclear. Another similar project is the 'Huggy Pyjamas' by Cheok [17] that exchanges hugs through pneumatic actuators, allowing stronger sensations, but on the cost of wearability.

Thecla Schiphorst has worked on developing 'Semantics of Caress' [18] that investigates how the meaning of touch can be applied to tactile interaction. This system represents touch and movement as something meaningful, contributing to quality sharing. Having identified intrinsic values of haptic communication in systems with relatively low haptic resolution, one of our research questions has been how this can be translated into functioning, wearable systems that produce a greater degree of tactile immersion? High fidelity haptics implies a haptic resolution of $90+$ effectors/ actuators [13].

Usability issues such as weight, volume and power consumption poses a serious challenge to future system designs of wearable, mobile haptic systems. Lindeman et al.'s research [19] on full-body haptic feedback through applications made with their 'TactaVest' haptic feedback system attempts to complete the user's sense of tactile immersion in a VR-based environment. The resolution of the early TactaVest physically confirms the haptic vision [5] experienced through VR. However, with only 8+ vibrotactile effectors it does not appear to have a high enough haptic resolution to provide a sense of sensory immersion on its own.

\footnotetext{
${ }^{2}$ As the number of stimulators over surface of stimulation.
} 


\section{Artistic Research Methodology}

The development of our haptic system has followed the path of affective interaction design where key aspects of the process are to effect emotional responses in the target user [20]. Emotional experiences do not solely reside in our minds or brains. They are experienced throughout our whole bodies [21]. Emotions have a crucial role in the human ability to understand and learn new things. Objects that are aesthetically pleasing appear to the user to be more effective by virtue of their sensual appeal [22].

Our research into affective haptics is grounded theoretically on practiced-based artistic research that is formed by the practice of making art [23]. Such artistically guided research is integrated in our projects through the construction of different practical-aesthetical experiments. Our various projects represent empirical research through testing prototypes of mobile, haptic interfaces. The advantages of building prototypes are many [24]. First of all it facilitates testing conditions that are not covered by established principles of design. Second, it provides an evaluation of a first concept for user interface as well as giving quick feedback from the user(s). Drawbacks include the temporary and limited experiential construction of prototypes. The scope of our prototypes has been to cover specific and aesthetically relevant aspects of technologically produced touch.

All our experiments have an open, explorative character, addressing the affective dimensions of haptic experience. How is this useful in scientific contexts? According to Schön, artistic works can be seen to represent knowledge, and the way the artist makes them reflects artistic methods [25]. We see making artworks as an integral part in building systematic knowledge about the use and application of haptic experiences. Our combination of affective interaction design approach with artistic exploration attempts to provoke emotional experiences in the user target group to reveal areas of problems throughout the different iterations of the hardware design.

Throughout our projects we have systematically applied a combination of the following methods: i) artistic practice-based research, ii) user interviews and iii) user observation. These were applied as tools to systematically create knowledge relevant to our goal of gaining insight and knowledge about haptic stimulus in mobile settings. The complex and multifaceted character of practical-aesthetical experiments demands bricolaged and interdisciplinary methods, therefore the use of 'hybrid methodology' is also suitable to describe our research

\subsection{First Generation Mobile Haptics}

Our first collaborative project, World Ripple, used GPS coordinates and satellite based navigation to create immaterial sculptures placed in the open outdoor landscape. The sculptures are either location based events, a kind of haptic theatre, or dynamic (data) structures moving, changing, developing their dimensions and properties over time' [26]. Users wore a bodysuit controlled by a GPS enabled laptop which connected to a micro-controller board and a custom made extension board called 
'the dusk' made for controlling the 64 coin-shaped vibrators inside the bodysuit. The bodysuit is worn underneath the ordinary clothing and the portable, sensor- and GPS based system is carried in a shoulder bag together with a laptop computer which controlled the entire system.

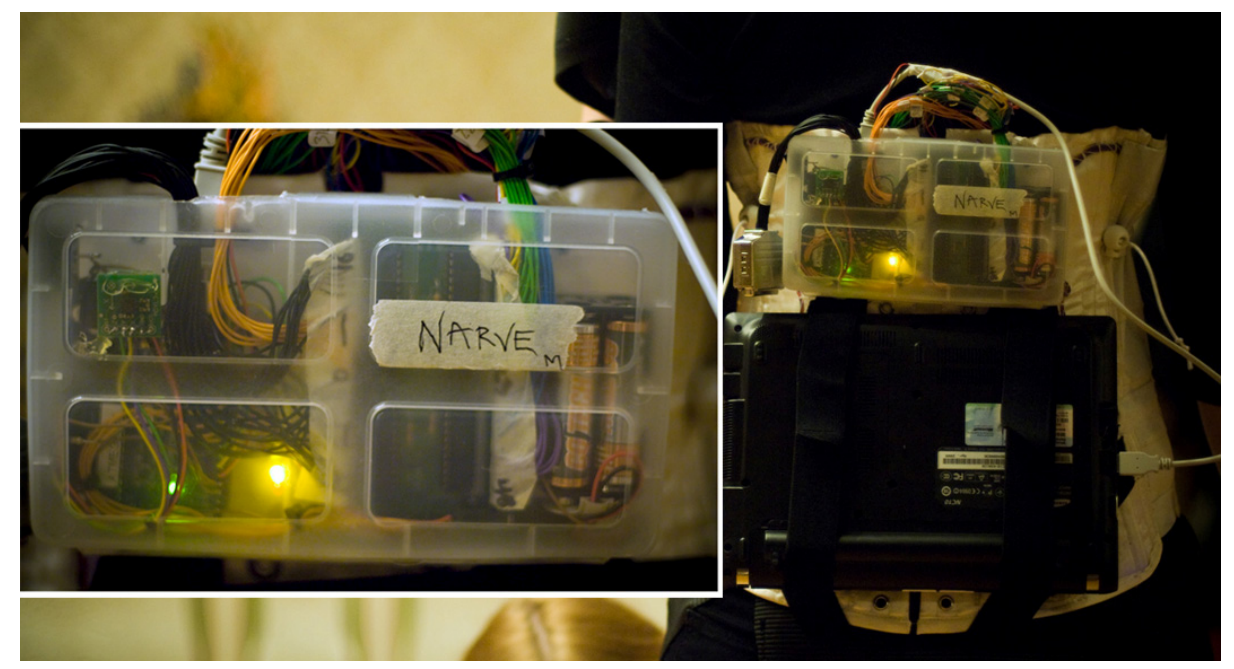

Fig. 1. Controller box with DUSK board (64 actuators) for BlindTheatre Bodysuit

The next iteration of the system was named 'The Blind Theatre' and turned the body into the stage of a somatic theatre (Fig. 1 and 2). It was performed indoors in 2009 at the Norwegian National Theatre in Oslo ${ }^{3}$. Users experienced an audio-haptic story while walking blindfolded around in the theatre. They reported high-order tactile percepts such as stroking, rubbing and caressing [27].

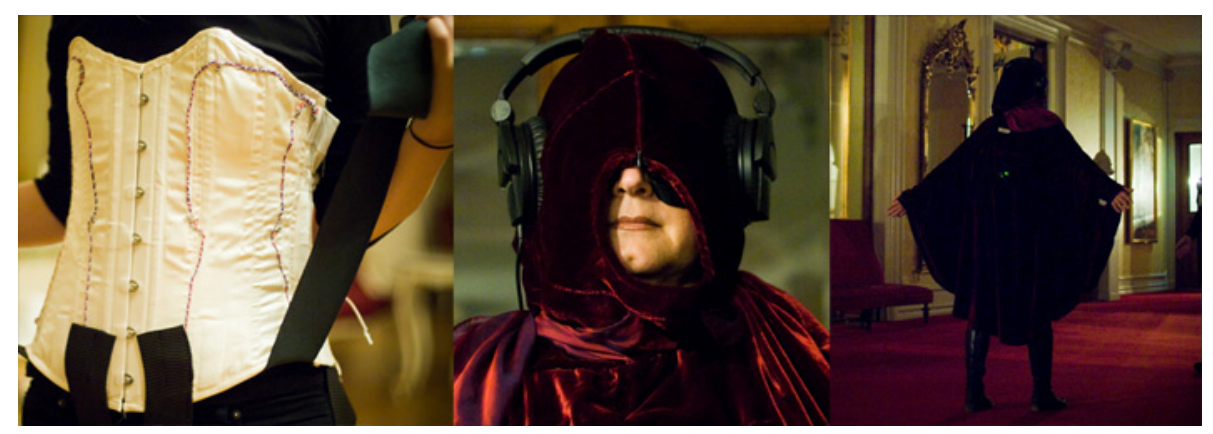

Fig. 2. The bodysuit for the Blind Theater. The haptic suit had two functional layers, one inner corsette and an outer, looser-fit cape.

${ }^{3}$ The blind theater project. http: / /blindtheater.wordpress. com 


\subsection{Second Generation Mobile Haptics}

The third iteration of the design replaced the laptop with the use of smartphones. This increased both comfort and maneuverability due to the smartphones weight and small form factor.

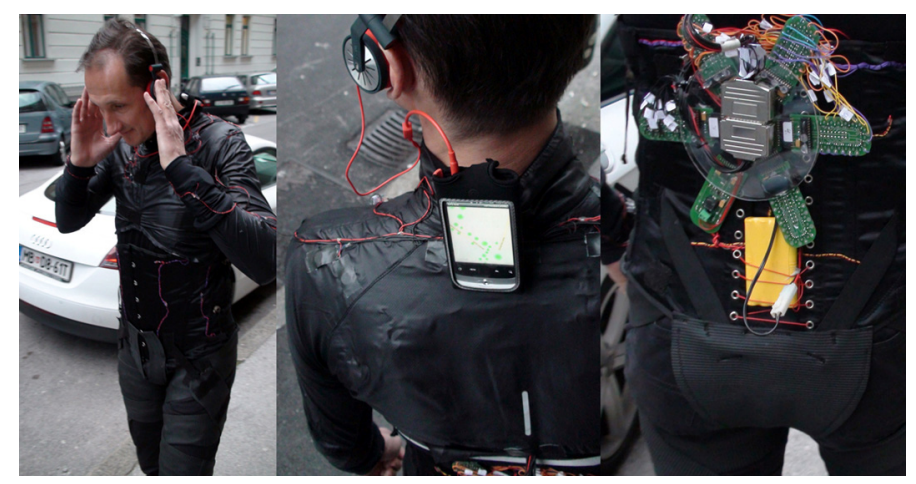

Fig. 3. The Psychoplastic bodysuit during use in Ljubljana 2010

Much like World Ripple this system used GPS to geotagg haptic data into zones defined by spatial coordinates. When entering predefined zones, users would trigger and sense the space through different combinations of vibrotactile patterns and sound (voice).

The phone both acted as GPS receiver and also ran the application controlling the vibrotactile outputs. The data was sent via Bluetooth to a custom made hardware named "Leaf" which consisted of a communication and control board extending into 5 boards shaped like flower leafs where each board controlled 16 vibrating motors, totaling 80 high-resolution actuators.

\subsection{The Sense Memory Experiment}

In the next Sense Memory experiment (2011) we simplified the system in order to experiment with an outdoor theatre involving multiple users. Constraints were cost and reliability. Using the new Arduino Mega board to Android system, the body suit contained only 16 high-resolution actuators. Although the haptic resolution was significantly lower, it simplified the first iteration of development and allowed us to prototype new hardware solutions within hours, focusing on maximizing the haptic effects outputs and design of haptic patterns. This also simplified the construction of the bodysuit, allowing us to - if needed - rapidly produce multiple suits.

Shaped as a cape (Fig. 4) the final bodysuit was designed for all-weather, outdoor use. Also here the user experienced invisible 'sculptures' by walking around in selected areas. New sets of haptic sculptures/expressions were geo-tagged onto 30+ zones placed around the square. Once the user enters one of the invisible geographic zones the sculptures came alive inside the cape as a combination of binaural sounds and vibrotactile patterns. Every zone contained different words and poetic expressions about emptiness in combination with unique haptic patterns. Here the user's walk formed combinations of touch and words into a unique somatosensory story. 


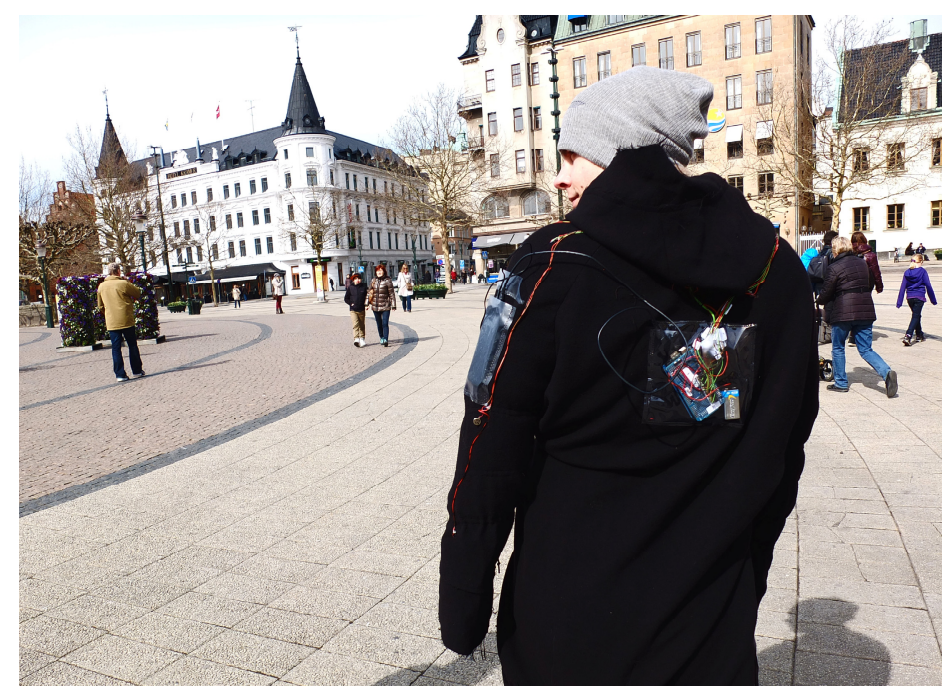

Fig. 4. The Sense Memory Cape during the Malmö test

\subsection{User Observation and Analysis}

As part of our artistic research user feedback were collected through conducting informal interviews with several participants throughout all the iterations of the system. In combination with our own observations we present the following analytical results:

1. Immersive closure of space: Walking around in the public square during normal daytime activities, users reported they were mindful of it beforehand, but once inside one of the suit they quickly immersed into the experience and forgot about possible onlookers. This indicates a closure of space, strengthening users' sense of an intimate, personal and 'inner' experience.

2. Multimodal strengthening of senses indicating the affective roles of haptics and audio in interaction: the cross-modal combination of sound and touch was experienced as intermingled, intertwined into a mutual strengthening of stimulus. Most users reported focusing mainly on the sound heard and that this appeared as the strongest stimulus. However, they also commented that the touches experienced made them stay longer, thus intensifying the overall sensation of body and space.

3. Increase of spatial awareness: a higher degree of spatial awareness was both observed and reported. Users wearing one of the systems noticeably changed their movement in space, becoming more aware of how they were moving to find both new and previous zones of experience.

4. Behavioral change: as users were free to move around in the open space we possibly expected a systematic, grid like search for the various interactive areas. However, once they entered the first interactive area they tended to move slowly before stopping. Thereafter they were observed to move in what can be described as an irregular, search like manner, moving back and forth, turning back to previous zones. The quick adaptation to new movement and behavior indicates how easily users can adapt to haptic technologies. 


\subsection{Discussion}

There are both benefits and problems with using an artistic exploration approach to the development of a wearable haptic system. Benefits compared to a traditional engineering approach that would focus on low level powering issues, long battery lives, or similar, is the artistic based strengthening of focus on the experiential dimension and user experience. Haptic and content related issues so become much more apparent in the early stages of development. In this way we can be more effective in creating valuable user experiences in faster design iterations. Such findings include positioning and repositioning of vibrotactile effectors in relation to sound and sequencing of haptic patterns. Focusing on users' sense of embodiment and immersion we found that anything less than 64 high-resolution haptic actuators seem to reduce the strength of the experience. When compared to other projects using higher densities of high resolution, vibrotactile stimulation such as Erotogod [13] a preferable number for a full bodysuit covering most parts of the body would be $90+$.

One major challenge is how to develop and design haptic patterns that allow for high-order tactile percepts. Although not treated specifically here, our projects have designed iterations of haptic editors that facilitate rapid coding and testing of advanced haptic patterns. In combination with our wide range of body suit designs this allows for optimal actuator placement and combinations on the body.

Other challenges connected to the use of an artistic exploration approach relate to the evaluation of user feedback. In the nature of interactive art projects, the user becomes an integral part of the emotional experience they are evaluating. Though observations were made on changes in behavioral patterns during tests, assuring their scientific relevance is difficult since the users could not, in most cases, confirm or deny these changes as they were both performing an art project and at the same time being the actual art piece.

\section{Conclusion and Future Developments}

This paper contributes to i) the construction of functional, wearable and haptic experience systems and ii) the discourse of how actual embodiment is experienced within human computer interaction. The combination of i) hardware development and ii) conceptual, aesthetical work has greatly helped us develop new scenarios and novel approaches to the field of haptics. Our field tests show how geotagging haptic experiences greatly affects the users embodied experiences such as sense of place. Another outcome from our experiments is the suggestion that the experience of full body haptic immersion need a haptic resolution of $90+$ effectors. There are many improvements to be made and future developments need to include high resolution and wearable prototypes capable of producing advanced haptic experiences for users on the move. Another aim is setting up a more rigid frame work for the evaluation of the artistic exploration approach to strengthen the academic relevance of the highly subjective results such artworks produce. 


\section{References}

1. Dourish, P.: Where the action is: The Foundations of Embodied Interaction. The MIT Press, Cambridge (2001)

2. Barnouw, E.: Mass communication: television, radio, film, press: the media and their practice in the United States of America. Rinehart, New York (1956)

3. Grau, O.: Virtual Art - From Illusion to Immersion. The MIT Press, Cambridge (2004)

4. Zielinski, S.: Archäologie der Medien. Rowohlt Taschenbuch Verlag (2002)

5. Paterson, M.: The Senses of Touch: Haptics, Affects and Technologies. Berg, Oxford (2007)

6. Fong, B., Fong, A.C.M., Li, C.K.: Telemedicine Technologies: Information Technologies in Medicine and Telehealth. John Wiley \& Sons, West Sussex (2011)

7. Dominjon, L., Perret, J., Lécuyer, A.: Novel devices and interaction techniques for humanscale haptics. The Visual Computer 23, 257-266 (2007)

8. Van Veen, H.A.H.C., Van Erp, J.B.F.: Providing Directional Information with Tactile Torso Displays. Presented at Eurohaptics 2003 (2003)

9. Weiser, M.: The computer for the 21st century. ACM SIGMOBILE Mobile Computing and Communications Review 3, 3-11 (1991)

10. York, J., Pendharkar, P.C.: Human-computer interaction issues for mobile computing in a variable work context. International Journal of Human-Computer Studies 60, 771-797 (2004)

11. Marion, A., Heinsen, E., Chin, R., Helmso, B.: Wrist instrument opens new dimension in personal information. Hewlett-Packard Journal (1977)

12. Nagel, S.K., Carl, C., Kringe, T., Märtin, R., König, P.: Beyond sensory substitution learning the sixth sense. Journal of Neural Engineering 2, 13-26 (2005)

13. Stenslie, S.: Virtual Touch - A study of the user and experience of touch in artistic, multimodal and computer-based environments. Oslo School of Architecture and Design, Oslo (2010)

14. Sommerer, C., Mignonneau, L.: Mobile Feelings -wireless communication of heartbeat and breath for mobile art. In: Proceedings of The 14th International Conference on Artificial Reality and Telexistence, ICAT (2004)

15. Ibid (Sommerer \& Mignonneau, 2004)

16. Seymour, S.: Fashionable Technology. Springer, Vienna (2001)

17. Cheok, A.D.: Art and Technology of Entertainment Computing and Communication. Springer, London (2010)

18. Schiphorst, T.: $\operatorname{soft}(\mathrm{n})$ : Toward a Somaesthetics of Touch. In: Proc. of the 27th International Conference Extended Abstracts on Human Factors in Computing Systems, pp. 2427-2438. ACM, Boston (2009)

19. Lindeman, R.W., Page, R., Yanagida, Y., Sibert, J.L.: Towards Full-Body Haptic Feedback: The Design and Deployment of a Spatialized Vibrotactile Feedback System. In: Proc. of ACM Virtual Reality Software and Technology (VRST), Hong Kong, China, pp. 146-149 (2004)

20. Picard, R.W.: Affective Computing. MIT Press, Cambridge (1997)

21. Davidson, R.J., Scherer, K.R., Goldsmith, H.H.: Handbook of Affective Sciences. Oxford University Press, New York (2002)

22. Norman, D.: Emotional Design. Basic Books, New York (2005) 
23. Biggs, M., Karlsson, H.: The Routledge Companion to Research in the Arts, p. 126. Taylor \& Francis (2010)

24. Stary, C.: Interaktive Systeme. Software Entwicklung und Software-Ergonomie. Vieweg Informatik/Wirtschaftsinformatik (1996)

25. Schön, D.: The Reflective Practitioner. Basic Books, New York (1983)

26. World Ripple, http: //www.stenslie.net/?page_id=85

27. Gibson, J.: The senses considered as perceptual systems. Houghton Mifflin, Boston (1966) 